\title{
Secured route optimization in mobile IPv6 wireless networks in terms of data integrity
}

\begin{abstract}
Route optimization (RO) in mobile IPv6 (MIPv6) provides a mobile node (MN) to communicate with correspondent node $(\mathrm{CN})$ directly, using shortest possible path and eliminate inefficient triangle routing. MIPv6 uses return routability procedure to authenticate and secure binding updates. There is no authentication and data protection method in RO when MN moving from one network to another, in RFC 3775, standard for MIPv6. In this paper, a new security method is proposed in terms of data integrity, which makes use of strong and light data encryption and using HA for key management and distribution purpose. An enhanced security algorithm is developed on top of MIPv6 RO to secure data and prepare safe communication between $\mathrm{MN}$ and $\mathrm{CN}$. This algorithm is able to detect and prevent the attacker from modifying the data with using an encryption algorithm at a cost of a small increase in delay. The real time network test-bed is implemented to prove the efficiency of proposed method. The experimental results show that the proposed security scheme increases the security performance of the network. This gives advantage of safe communication that can significantly improve the data security in RO while maintaining the quality of other network performance.
\end{abstract}

